\title{
1. Clumped Dispersal and Species Coexistence
}

\section{Clumped Dispersal and Species Coexistence}

\author{
Authors: \\ Michael Potthoff ${ }^{1}$ mpotthoff@awi-bremerhaven.de \\ Karin Johst $^{2} \quad$ karin.johst@ufz.de \\ Julian Gutt ${ }^{1} \quad$ igutt@awi-bremerhaven.de \\ Christian Wissel $^{2}$ \\ ${ }^{1}$ Alfred Wegener Institute for Polar and Marine Research, \\ Columbusstr, \\ 27568 Bremerhaven, Germany \\ ${ }^{2}$ Department of Ecological Modelling, \\ UFZ Centre for Environmental Research Leipzig-Halle, \\ PO Box 500135, \\ 04301 Leipzig, Germany
}

This article has been published in

Ecological Modelling

Volume 198, Issues 1-2, 15 September 2006, Pages 247-254

doi:10.1016/j.ecolmodel.2006.04.003

The original article is available at http://www.sciencedirect.com/

This is an author-created version of the unchanged article according to the copyright agreement between the authors and the publisher. 


\subsection{Abstract}

Anisotropic clumped (patchy) dispersal of species is a widespread phenomenon in nature, however its relevance for structuring communities and maintaining biodiversity is still a matter of debate. We used a spatially explicit simulation model to assess the effects of spatial aggregation through this dispersal strategy compared to isotropic clumped (local) dispersal on the diversity of a model community of sessile species. The species differed only in terms of their dispersal distance and no a priori trade-offs between dispersal and local competitive strength were assumed. We investigated spatially homogeneous and heterogeneous environments without and with temporally fluctuating reproduction success. In all cases patchy dispersal allowed the long-term coexistence of a variety of species - in contrast to local dispersal. In particular, patchy dispersal was found to decouple the mechanism of spatial aggregation from the dispersal distance. This supports species coexistence even in environments where local dispersal was a superior or inferior dispersal strategy. Thus, the specific way of spatial aggregation can be decisive for coexistence. Spatial aggregation independent of the dispersal distance is an attribute of dispersal offering new possibilities for trade-offs with life-history traits and with the dispersal distance itself. 


\subsection{Introduction}

Much theoretical research has been done to reveal and understand the mechanisms that lead to the coexistence or to the exclusion of species and subsequent succession. Dispersal is one of the major factors shaping communities and mediating coexistence. Field observations show that dispersal patterns can be highly complex. Most marine species for example disperse through pelagic larvae stages (Roughgarden et al., 1988; Muko and Iwasa, 2000). Since they are exposed to the same environmental influences and originate simultaneously from a specific location, they often behave as a swarm and disperse as a group (Hofmann et al., 1998; Flierl et al., 1999; Lockwood et al., 2002). This will result in an anisotropic and clumped (patchy) dispersal pattern. Such patchy dispersal seems to be a widespread phenomenon in nature, not restricted to marine environments (David et al., 1997; Heard and Remer, 1997; Smith and Witman, 1999; Nathan and Muller-Landau, 2000; Wenny, 2001). It can be generated by various abiotic or biotic dispersal agents (wind and water currents, animals) or a combination thereof (Nathan and Muller-Landau, 2000; Stoyan and Wagner, 2001; Wenny, 2001; Wang and Smith, 2002; Wagner et al., 2004). Using abiotic dispersal agents, landscape elements can act as an attractor or interceptor for dispersal stages (Reed et al., 2000) like any kind of obstacle, e.g. a scrub in an open landscape or a rock in the sea. Using biotic dispersal agents, patchy dispersal can emerge due to the behaviour of the agent (Heard and Remer, 1997; Wenny and Levey, 1998; Fragoso and Huffman, 2000; Hartley and Shorrocks, 2002; Wehncke et al., 2003). Secondary dispersal processes such as scatter hoarding may also be an important source of patchy dispersal (Nathan and MullerLandau, 2000; Wang and Smith, 2002; Roth and Vander Wall, 2005). Notably, fertilization success of sedentary organisms like wind-pollinated plants or sessile marine animals is greatly influenced by anisotropic gamete dispersal as well (Claereboudt, 1999).

Theoretical approaches modelling dispersal, however, often assumed isotropic global dispersal (mean-field assumption) where all units can freely interact over all scales. This is appropriate as long as suitable habitat is abundant (King and With, 2002). However, as Durrett and Levin (1998) point out, it neglects spatial aspects and thus can have important consequences for diversity (Murrell et al., 2001). More realistic approaches distribute dispersal stages according to particular dispersal kernels over certain distances but assume the adult at the centre and dispersal of individual 
propagules or offspring being possible in all directions (Clark et al., 1999; Hovestadt et al., 2000; Levin and Muller-Landau, 2000; Nathan and Muller-Landau, 2000; Johst et al., 2002). This isotropic (around the source) and local dispersal may lead to local clustering and has a positive effect on diversity when there is a competitive difference among the species with respect to favourable habitats (Chesson, 1994; Chesson and Neuhauser, 2002; Snyder and Chesson, 2003).

Modelling a realistic seed abscission process for anemochorous plants Schippers and Jongejans (2005) demonstrated that an anisotropic seed shadow can emerge, that strongly influences the long distance dispersal which in turn is essential for maintaining diversity (Higgins et al., 2003). A theory linking such anisotropic dispersal and diversity is the aggregation model of coexistence (Shorrocks et al., 1979; Hartley and Shorrocks, 2002). This theory has been used to explain the high diversity of insect communities utilising ephemeral resources. It states that individuals of a species may be clumped together in a way limiting their own population growth such that resources that are left free can be utilised by inferior competitors (Hartley and Shorrocks, 2002). Thus, the basic mechanisms are the well known coexistence criteria "intraspecific competition higher than interspecific competition" (see e.g. Begon et al. 1998; Murrell et al.2001) and dispersal limitation of a superior competitor (Hurtt and Pacala, 1995). These mechanisms also play a role in the so-called competition-colonisation trade-off mechanism (Tilman, 1994 but see Higgins and Cain, 2002). It assumes that in a spatially structured population the locally superior competitor is the inferior disperser, e.g. has the shorter dispersal distance.

All these mechanism relate diversity to dispersal and have been found to work in theory as well as having been demonstrated in nature. However, they are based on species difference and spatial heterogeneity to work and cannot explain the coexistence of apparently similar species in homogeneous environments. For example, it is unclear whether coral reefs or rain forests provide enough niches to explain the high diversity in these habitats. The unified neutral theory (Hubbell, 2001) tries to fill this gap. One major assumption of this theory is that niches are absent and extinction, immigration and speciation are the essential processes structuring the communities on evolutionary time scales (Hubbell, 2001; Volkov et al., 2003). Dispersal influences the long-term structure of a neutral community via its influence on the extent of species mixing. Although neutral models are able to reproduce species-area relationships surprisingly well (Hubbell, 2001; Chave, 2004; McKane et 
al., 2004) even slight violations of the neutrality can lead to a quick breakdown (Fuentes, 2004). Therefore, the neutral theory has been controversially discussed (Nee and Stone, 2003; Adler, 2004; Alonso and McKane, 2004).

In general four forms of dispersal may be distinguished: a) global dispersal (isotropic non-clumped), b) local dispersal (isotropic clumped), c) directed dispersal (anisotropic, e.g. along a vector) and d) patchy dispersal (anisotropic clumped). Dispersal kernels of the type a) and b) have been extensively studied as the isotropic approach has the advantage of simple analytical tractability.

Directed dispersal (type $\mathrm{c}$ ) has been found to affect the diversity and genetic flow of populations (Wenny, 2001) and spatial heterogeneity in sedentary organisms (Reed et al., 2000). Wagner et al. (2004) modelled anisotropic seed dispersal using a cluster point process and proposed a positive effect for long distance disperser (e.g. pioneer species) when suitable habitat is rare.

More complicated dispersal kernels (e.g. type d) and the consideration of spatially and temporally variable environments (which can influence the competitive advantage of a dispersal strategy) complicate analytical tractability. Simulation models provide an alternative way to investigate such complex situations. Some attempts have been made using correlated random walk or Lagrangian models (Yamazaki and Haury, 1993; Yamazaki and Okubo, 1995). However, these explicit methods are time consuming and not well suited for high individual numbers. Therefore, simplified approaches are needed when analysing the influence of complex dispersal pattern on diversity.

In the following we will focus on patchy (anisotropic clumped) dispersal. To our knowledge and compared to type b) dispersal, its relevance for species coexistence has not yet been investigated. What are the characteristically properties of such a type d) dispersal mechanism? Although patchy dispersal results in a high number of propagules at some sites, thus creating spatial aggregation similar to local dispersal, the peak propagule density is not at the original site. Therefore, patchy dispersal bears aspects of both local and global dispersal and it is not obvious which aspects dominate in different environments and competitive scenarios. For that reason we investigate the effects of patchy dispersal on diversity (in the sense of species richness) with a simulation model for neutral (with respect to demographic parameters) and hierarchical communities in different environments. We demonstrate that patchy dispersal can promote coexistence in heterogeneous as well as in 
homogeneous environments and between competitively equal and competitively different species. Thus we show that patchy dispersal is able to mediate coexistence even in situations in which local dispersal is unable for it. Although we use the terms 'adult' and 'larva', these terms can be replaced by others such as tree and seed, as our model is general and can describe many sessile and sedentary organisms in many kinds of habitats.

\subsection{The model}

The model space is represented by a rectangular, two-dimensional grid of $50 \times 50$ cells. To avoid boundary effects we use periodic boundary conditions, i.e. simulation is performed on a torus around which all the borders of the grid are warped. Each cell is equivalent to a site which can support a total number of individuals corresponding to the local carrying capacity $K_{C}$ of the site.

\subsubsection{Homogeneous versus heterogeneous environments}

If all the sites have the same local carrying capacity, we refer to a homogenous environment. If the local carrying capacity changes from site to site we refer to a heterogeneous environment. For simplicity, we distinguish only two different site types: "high capacity sites" where $K_{C}=K_{H}$ and "low capacity sites" where $K_{C}=K_{L}$. In such a heterogeneous environment the carrying capacity of each site is randomly chosen with the probability $p$ of being $K_{H}$ and $1-p$ of being $K_{L}$ and remains constant during a simulation.

\subsubsection{Species definition}

To focus on the effects of the dispersal pattern on diversity, all the species in our model have the same competitive strength and mean reproductive rate (number of larvae, see constant vs. fluctuating environment below). We do not assume any trade-offs or disturbance events. Differences in the species traits consist in the mean dispersal distance $D_{s}$ before settlement (see below). As we consider sessile species, we assume larvae to be the only dispersal stages. Further on, we distinguish 
between two dispersal modes: local ("isotropic clumped dispersal") and patchy ("anisotropic clumped dispersal”). The modes are described below in more detail.
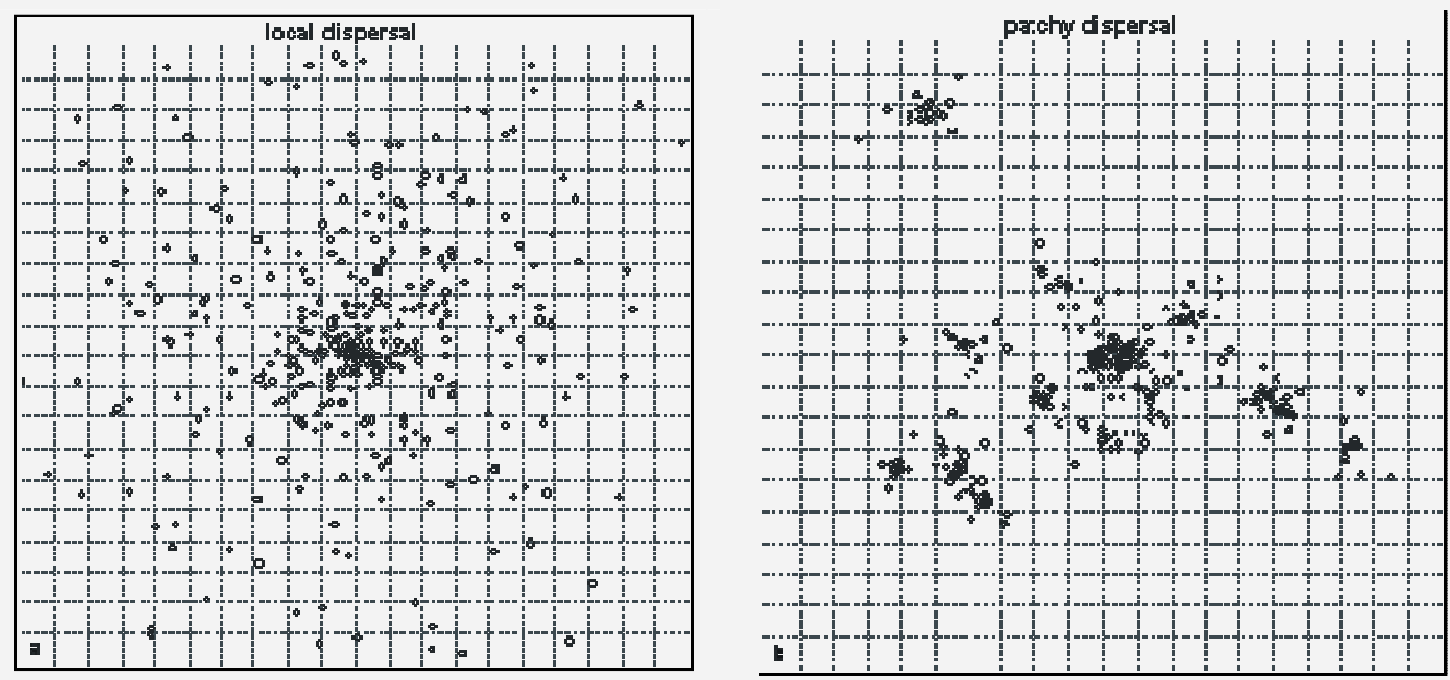

Figure 1, Examples of the spatial dispersal pattern generated by the different dispersal strategies. a) local ( isotropic clumped) dispersal, b) patchy (anisotropic clumped) dispersal. Each picture shows the final larvae pattern after the dispersal of $20 \times 20$ larvae originating from the centre.

\subsubsection{Local dispersal}

Commonly used dispersal kernels are exponential or Gaussian distributions (Nathan and Muller-Landau, 2000). In our model the direction a of dispersal was randomly chosen $\left(0^{\circ}-359^{\circ}\right)$ and the actual distance $d$ was determined according to $d=\mathrm{D}_{S}{ }^{*} \operatorname{In}(p)$ with $p$ being a random number evenly distributed in the interval [0..1]. The Larva settles at the position given by the relative polar coordinate $P(d, \alpha)$. An example of a resulting larvae distribution pattern emerging from this dispersal mode is shown in Figure 1a.

\subsubsection{Patchy dispersal}

Here all the larvae of a species at a particular site are considered as a group. For this group the centre of dispersal is chosen using the same dispersal kernel as above. Then, all the larvae of this group are distributed around this centre according to a 
kernel with a perimeter of 2 cells. This results in a patchy aggregation of larvae. The resulting dispersal pattern can be seen in Figure 1b.

\subsubsection{Local dynamics}

In each time step an adult individual of any species produces $L$ larvae (see below). After reproduction larvae dispersed, resulting in a local larvae pool of different species for each cell (see dispersal). Adult individuals die with a fixed probability $p_{d}=0.2$ resulting in free positions in a cell. All larvae in a local larvae pool compete for empty positions described by lottery competition. For each empty position a larva is randomly taken from the local pool, which reduces the number within the pool. This is repeated until either the number of established individuals reaches $K_{C}$ or there are no larvae left. Larvae that are unable to establish are discarded.

\subsubsection{Constant versus fluctuating environments}

The carrying capacities of the grid cells remained fixed in all cases. Environmental fluctuations are assumed to affect the reproduction success of established individuals. Therefore $L$ was set to $L=1$ and not modified in constant environments. In fluctuating environments $L$ was randomly chosen to be $L=0, L=1$ or $L=2$ for each cell and in each time step.

\subsubsection{Initial conditions}

The first quarter of the grid was initially filled with a random community consisting of all available species. For each cell in this area, one of the species was randomly assigned and occupied all the available space in this cell. All simulations were allowed to run either until a certain final time or were stopped when either all species had gone extinct or just one had survived. In both cases it was assumed that the species composition would not change again until the final time step was reached. 


\subsection{Computer simulation experiments}

In the first experiment we implemented a neutral model to see if there is a general difference between local and patchy dispersal. We assumed 15 species with identical traits all using firstly local dispersal and secondly patchy dispersal. The species had an intermediate dispersal distance $\left(D_{S}=5\right)$. In this experiment we assumed both homogenous $\left(K_{H}=5, K_{L}=5\right)$ and heterogeneous $\left(K_{H}=7, K_{L}=3\right.$ and $\left.p=0.5\right)$ space. The experiment ran until time step $1.5^{\star} 10^{5}$ with 200 replicate runs.

In a second experiment we again assumed 15 species but now with different dispersal distances ranging from 1 to 15 cells in steps of 1 . As shown by Hovestadt et al. (2000) this reflects different competitive abilities of the species. In this experiment we used the same environmental scenarios like in the first experiment. Additionally, we tested both temporally constant and fluctuating environments. Maximal runtime was $2^{*} 10^{5}$ time steps and we computed 200 replicates

The first two experiments concentrated on the impact of patchy and local dispersal on community structure separately, that is all species used either local or patchy dispersal. In the third experiment we studied the competition between these two dispersal modes. First the local dispersal was rather short-ranged $\left(D_{S}=2\right)$ and the patchy dispersal was long-ranged $\left(D_{S}=10\right)$, then the dispersal distances were exchanged. To account for the influence of spatial heterogeneity we used a gradient from homogeneous to heterogeneous space by setting $K_{H}=\mathrm{i}$ and $K_{L}=10-K_{H}$ with i running from 5 to 10 and $p=0.5$ such that the total amount of habitat was equal for all simulations. Again we tested temporally constant and fluctuating environments. Due to the low species number maximal runtime was set to $0.75^{\star} 10^{5}$ time steps and 100 replicate runs. 


\subsection{Results}

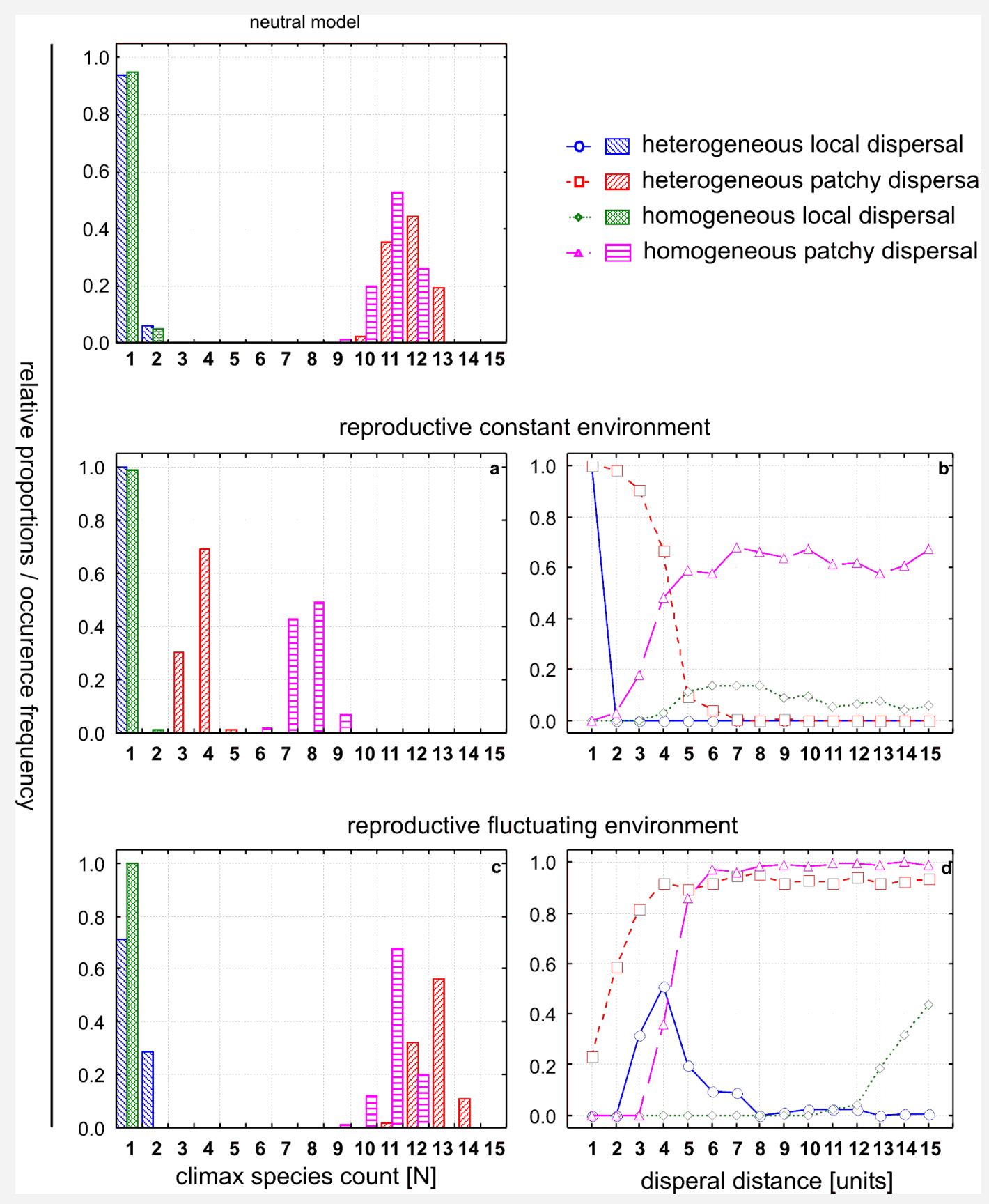

Figure 2, Species count and survival of single species, depending on different environments and species traits. Left: probability ( $y$-axis) of $n$ species ( $x$-axis) survival after 100 replicate runs $\left(T_{\max }=2^{*} 10^{5}\right)$. Right: probability of survival until the climax is reached ( $y$-axis) for species $i(i=1-15)$. 


\subsubsection{Experiment 1 (neutral model)}

Figure 2 shows the proportions of species in the climax stage. Nearly all runs with local dispersal ended in single species communities (Figure 2). Mean convergence time $\Delta T_{C}$ into a single species system was $\Delta T_{C}\left(D_{S}=5\right.$,hom $) \sim 0.67^{*} 10^{5}( \pm 0.28)$ and $\Delta \mathrm{T}_{\mathrm{C}}\left(\mathrm{D}_{S}=5\right.$, het $) \sim 0.66^{*} 10^{5}( \pm 0.28)$ time steps. Simulations with local dispersal and different dispersal distances showed that $\Delta \mathrm{T}_{\mathrm{C}}$ could be described by a power law: $\Delta \mathrm{T}_{\mathrm{C}}(\mathrm{hom}) \sim 0.89 * 10^{5 *} D_{S}^{-0.084}\left(\mathrm{r}^{2}=0.41\right)$ and $\Delta \mathrm{T}_{\mathrm{C}}(\mathrm{het}) \sim 0.86^{*} 10^{5 *} D_{S}^{-0.269}\left(\mathrm{r}^{2}=0.81\right)$. See also Figure 3 for a comparison of the species extinction speed. Assuming patchy dispersal, $10-13$ species were able to coexist at least for $1.5{ }^{*} 10^{5}$ time steps.

\subsubsection{Experiment 2 (different mean dispersal distances, single dispersal mode)}

Figure $2 \mathrm{a}$ shows that neither of the local dispersal strategies led to a notable coexistence of several species. In contrast, the patchy dispersal strategies led to the coexistence of several species in all cases: In a constant environment this dispersal strategy allowed 7-9 species (homogeneous environment) or 3-4 species (heterogeneous environment) to coexist. In a fluctuating environment even more species coexisted: about 10-12 in the homogeneous and 12-14 in the heterogeneous environment (Figure 2c). In the homogeneous environment the surviving species were intermediate to long-range dispersers (Figure 2b). In the heterogeneous environment short-range dispersers (Figure 2d). The clear ranking of abundance according to dispersal distance in the heterogeneous environment was not found in the homogeneous environment albeit the general failure of short range dispersers.

\subsubsection{Experiment 3 (different mean dispersal distances and two dispersal modes)}

Depending on the environmental conditions local or global dispersal could coexist with patchy dispersal. Table 1 lists the relative abundances of both dispersal strategies in different environments and different degrees of spatial heterogeneity. In constant environments coexistence between global long- and patchy short-range dispersal was possible when space was sufficiently heterogeneous. In reproductively fluctuating environments coexistence between these dispersal modes was possible under strong spatial heterogeneity. Coexistence between local short- and patchy long-range dispersal was possible when space was homogeneous. 


\begin{tabular}{|c|c|c|c|c|c|c|c|c|c|c|c|c|c|c|}
\hline \multirow{3}{*}{$\begin{array}{l}\text { environment } \\
\text { constant }\end{array}$} & \multicolumn{2}{|c|}{$\begin{array}{l}\text { competing dispersal strategies } \\
\text { local / patchy }\end{array}$} & \multicolumn{12}{|c|}{ mean relative abundance after $0.75^{\star} 10^{5}$ time steps [ $\left.\mathrm{N}=100\right]$} \\
\hline & long & short & 1.00 & & 1.00 & & 1.00 & & 0.88 & 0.12 & 0.79 & 0.21 & 0.71 & 0.29 \\
\hline & short & long & 1.00 & & 1.00 & & 1.00 & & 1.00 & & 1.00 & & 1.00 & \\
\hline \multirow{2}{*}{ fluctuating } & long & short & 1.00 & & 1.00 & & 1.00 & & 1.00 & & 1.00 & & 0.93 & 0.07 \\
\hline & short & long & 0.72 & 0.28 & 0.79 & 0.21 & 0.97 & 0.03 & 1.00 & & 1.00 & & 1.00 & \\
\hline \multicolumn{3}{|c|}{ heterogeneity (high:Iow capacity) } & $5: 5$ & & $6: 4$ & & $7: 3$ & & \begin{tabular}{|l|l|}
$8: 2$ \\
\end{tabular} & & $9: 1$ & & 10:0 & \\
\hline
\end{tabular}

Table 1, Relative abundances of competing dispersal strategies after $0.75^{*} 10^{5}$ time steps [ $\mathrm{N}=100]$. Depending on spatial heterogeneity and favourable dispersal distance, coexistence between isotropic and anisotropic dispersal is possible. Scenarios where coexistence occurred are shaded in grey. 


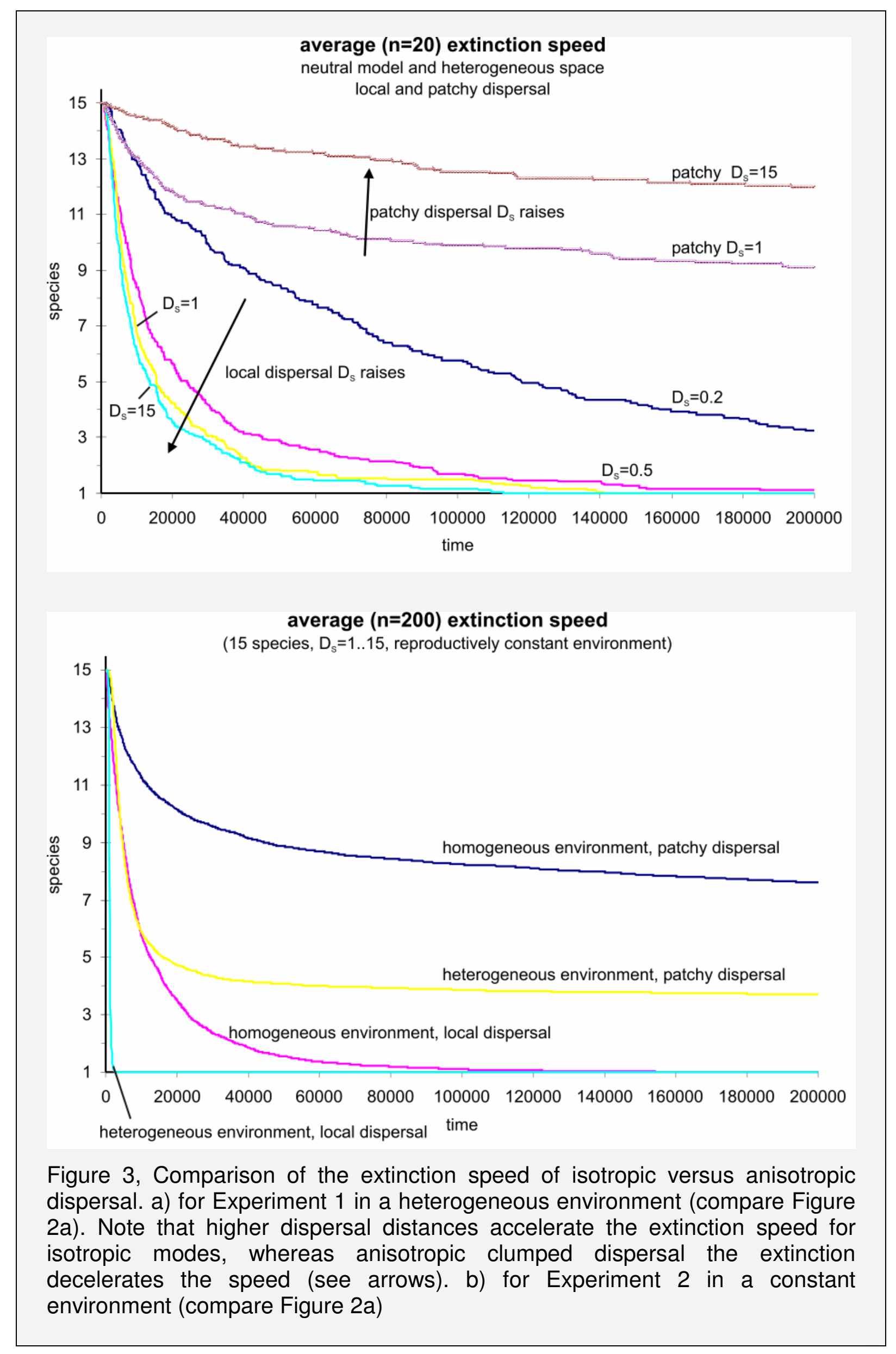




\subsection{Discussion}

Although patchy dispersal is known from field observations (e.g. dispersal as a group of larvae), previous modelling approaches considered mainly local dispersal as source of clustering in the context of species coexistence (e.g. Warren and Topping 2004). As both dispersal modes cause spatial aggregation, the comparison between patchy and local dispersal in terms of their potential to mediate coexistence is especially interesting and helpful for understanding coexistence mechanisms.

In our simulation experiments, patchy dispersal resulted in species-rich communities even under conditions that resulted in single species systems when local dispersal was assumed. This was true both within the neutral model assumption (experiment 1; for this assumption see also Chave, 2004) and the successional model assumption (experiment 2; for this assumption see also Hovestadt et al. 2000).

In contrast to local dispersal, patchy dispersal enabled the long-term coexistence of $1 / 4$ or up to $3 / 4$ of all competing species (Figure $2 a, c$ ). Note that the exclusion of species is due to stochastic events in the neutral model, but due to competitive inferiority (based on the different dispersal distances) in the successional model. These competitive differences also explain the different likelihoods to occur in the climax community for the different dispersal distances respectively species (Figure $2 \mathrm{~b}$ and $\mathrm{c}$ ). The associated dispersal distances of coexisting species tally with theoretical studies on the evolution of dispersal. In this context spatial heterogeneity discourages dispersal, i.e. species with patchy short-range dispersal coexisted in spatially heterogeneous environments (Figure $2 b$ ), whereas spatial homogeneity or fluctuating reproduction encourages dispersal (Hamilton and May, 1977; Hovestadt et al., 2000), i.e. species with patchy long-range dispersal coexisted in these environments (Figure 2d). The advantage of particular dispersal distances reflects the fact that patchy dispersal can partially utilize the competitive advantages of the appropriate dispersal distance in the corresponding environment but that there are additional mechanisms preventing the collapse into a single-species system.

In general, neutral models can be expected to show random extinction of all but one species (Tilman, 1994; Hubbell, 2001). If a species by chance becomes rarer, it will have fewer opportunities to win in the next time step and so forth as all species are assumed to be equal. Hence its abundance is spiralling down to extinction and coexistence is non-stable sensu Chesson (2000 a). Figure 3a exemplifies the extinction speed of a neutral community of 15 species with identical traits in a 
heterogeneous environment for different dispersal modes and distances. With isotropic dispersal higher dispersal distances lead to higher species mixing, thus stronger interspecific competition and consequently a faster extinction. Interestingly with patchy dispersal increasing dispersal distances decelerate the extinction process. In order to enable similar coexistence times, local dispersal would need to be extremely local (e.g. "clumped", $D_{S}<0.1$, i.e. virtually no dispersal). The effects of patchy dispersal are able to slow down the random exclusion of species in a neutral community to such an extent that it virtually allows coexistence up to evolutionary timescales (approximated e.g. for the homogeneous environment about $3.7^{\star} 10^{12}$ time steps, Figure 3).

The main difference between local and patchy dispersal is that patchy dispersal decouples the area where the larvae compete from the adult individual whereas local dispersal ensures that this area is always centred around the adult. Thus patchy dispersal decouples the larvae aggregation from the source. This has several important consequences.

First, similar to the aggregation model of coexistence (Shorrocks et al., 1979) the concentration of larvae leaves some sites free which can be utilized by other competitors. This concentration of conspecific competitors represents a strong dispersal limitation and has a stabilizing effect as it increases intraspecific relative to interspecific interactions (Chesson, 2000 a).

Furthermore, local aggregation can cause competitive displacement due to a 'phalanx growth' mechanism as demonstrated by Bolker and Pacala (1999). This mechanism is based on the exclusion of heterospecifics due to high self-recruitment, allowing a single species to seize habitats and displace competitors. In order to work, this process demands either a high number of larvae continuously placed around an adult (e.g. by local dispersal) or competetive differences. As patchy dispersal decouples the site where larvae compete from their origin, there is no continuous rain of larvae at the same place and consequently no high rate of self-recruitment. Therefore patchy dispersal hinders a phalanx-growth mechanism and weakens the displacement of species.

This effect is similar to the effect of positive growth-density covariance Chesson and colleagues found for intraspecific aggregation due to local dispersal under the presence of favourable habitats for particular species (Chesson, $2000 \mathrm{~b}$; Chesson and Neuhauser, 2002; Snyder and Chesson, 2003). Such a covariance occurs when 
limited dispersal allows rare species to build up densities in favourable areas, resulting in an increase of their overall per capita growth (Amarasaekare, 2003). It measures the degree to which competing species can accumulate in favourable locations (Snyder and Chesson, 2004). The key process for a positive growth-density covariance is a dispersal strategy which permits the establishment of a group of new individuals in suitable environments. This can be the result of local aggregation of larvae around an adult due to local isotropic dispersal in environments with species specific favourable habitats (Chesson, 2000 b; Amarasaekare, 2003; Snyder and Chesson, 2003). However, this can also be the result of a dispersal strategy where the aggregation of larvae causes temporarily suitable habitats due to the absence of competitors. Thus it depends in principle neither on local dispersal nor on species specific favourable habitat but solely on the aggregation of larvae at a certain suitable place.

These mechanisms also work in the successional model, where species have competitive differences and hierarchies caused by different dispersal distances (Hovestadt et al., 2000). With isotropic dispersal, only one species could survive (e.g. the local disperser in spatially heterogeneous constant environments or the global disperser in homogenous fluctuating environments (Figure $2 \mathrm{c}$ and e), which tallies with other theoretical studies (Hamilton and May, 1977; Hovestadt et al., 2000). With patchy dispersal, coexistence of several species was possible. An interesting result is that more species could coexist in the homogeneous than in the heterogeneous environment irrespective of the temporal fluctuations (Figure $2 \mathrm{~b}, \mathrm{~d}$ ). As in the homogeneous environment all patches are equal, short dispersal distances are not favoured and the competitive exclusion is weaker. Consequently more species are able to coexist. The same is true with respect to long dispersal distances in environments with fluctuating reproduction success.

Coexistence between local and patchy dispersal in competition was only possible when patchy dispersal had the superior dispersal distance in the corresponding environment, similar to the classic colonisation-competition trade-off (Chave et al., 2002; Amarasaekare, 2003; Kneitel and Chase, 2004). The reason is that isotropic dispersal is a better strategy to search an area for favourable habitats. Such dispersers spread their larvae broadly such that sites which are free of competitors (e.g. due to patchy dispersal) can be quickly colonized. 
Given these advantages of isotropic dispersal, why should anisotropic (patchy) dispersal exists in nature? Introducing additional dispersal traits such as the batch size and the dispersal patch radius, patchy dispersal allows a variety of further tradeoffs both within the dispersal traits themselves and with respect to other life-history traits. For example, it may be much more effective to protect a group of larvae or seeds in one dispersing unit like a capsule or cone than to protect a single seed with the same effect.

Frequency-dependent predation may also discourage isotropic dispersal, as it leads to a more frequent predator-prey encounter probability (Gendron, 1987). Schooling is a common strategy to minimize an individual's risk of falling prey, and similar effects can be assumed for patchily distributed larvae.

Finally, it should be questioned whether ideal isotropic dispersal is possible in nature as most habitats are subject to some kind of directional phenomena (Levine, 2003). The potential of patchy dispersal to foster species coexistence demonstrates that dispersal strategies and the subsequent spatial pattern can greatly influence species richness in communities. Our results suggest that besides the dispersal distance, the variety of other dispersal traits offers far more possibilities for spatial aggregation and thus for species coexistence than presently considered and investigated.

\section{Acknowledgments}

We would like to thank S. I. Higgins and I. Schewe for their valuable comments which helped us to improve the manuscript. 


\subsection{Literature cited:}

Adler, P.B., 2004. Neutral models fail to reproduce observed species-area and species-time relationships in Kansas grasslands. Ecology, 85: 1265-1272.

Alonso, D. and Mckane, A.J., 2004. Sampling Hubbell's neutral theory of biodiversity. Ecol. Lett., 7: 901-910.

Amarasaekare, P., 2003. Competitive coexistence in spatially structured environments: A synthesis. Ecol. Lett., 6: 1109-1122.

Begon, M., Harper, J.L. and Townsend, C.R., 1998. Ökologie. Spektrum, Heidelberg Berlin.

Bolker, B.M. and Pacala, S.W., 1999. Spatial moment equations for plant competition: Understanding spatial strategies and the advantages of short dispersal. Am.Nat., 153: 575-602.

Chave, J., 2004. Neutral theory and community ecology. Ecol. Lett., 7: 241-253.

Chave, J., Muller-Landau, H.C. and Levin, S.A., 2002. Comparing classical community models: Theoretical consequences for patterns of diversity. Am. Nat., 159: 1-23.

Chesson, P., 1994. Multispecies competition in variable environments. Theor.Popul.Biol., 45: 227-276.

Chesson, P., 2000 a. Mechanisms ofmaintenance of species diversity. Annual Review of Ecological and Evolutional Systems, 31: 343-366.

Chesson, P., 2000 b. General theory of competitive coexistence in spatially-varying environments. Theor. Popul. Biol., 58: 211-237.

Chesson, P. and Neuhauser, C., 2002. Intraspecific aggregation and species coexistence. Trends Ecol.Evol., 17: 210-211.

Claereboudt, C., 1999. Fertilization success in spatially distributed populations of benthic free-spawners: A simulation model. Ecol. Model., 121: 221-233.

Clark, J.S., Silman, M., Kern, R., Macklin, E. and Hillerislambers, J., 1999. Seed dispersal near and far: Patterns across temperate and tropical forests. Ecology, 80: 1475-1494.

David, P., Berthou, P., Noel, P. and Jarne, P., 1997. Patchy recruitment patterns in marine invertebrates: A spatial test of the density-dependent hypothesis in the bivalve spisula ovalis. Oecologia, 111: 331-340.

Durrett, R. and Levin, S., 1998. Spatial aspects of interspecific competition. Theor. Popul. Biol., 53: 30-43.

Flierl, G., Grünbaum, D., Levin, S.A. and Olson, D., 1999. From individuals to aggregations: The interplay between behaviour and physics. J. Theor. Biol., 196: 397-454.

Fragoso, J.M.V. and Huffman, J.M., 2000. Seed-dispersal and seedling recruitment patterns by the last neotropical megafaunal element in Amazonia, the tapir. J. Trop. Ecol., 16: 369-385.

Fuentes, M., 2004. Slight differences among individuals and the unified neutral theory of biodiversity. Theor. Popul. Biol., 66: 199-203.

Gendron, R.-P., 1987. Models and mechanisms of frequency-dependent predation. Am.Nat., 130: 603-623.

Hamilton, W.D. and May, R.M., 1977. Dispersal in stable habitats. Nature, 269: 578581.

Hartley, S. and Shorrocks, B., 2002. A general framework for the aggregation model of coexistence. J. Anim. Ecol., 71: 651-662. 
Heard, S.B. and Remer, L.C., 1997. Clutch-size behaviour and coexistence in ephemeral-patch competition models. Am. Nat., 150: 744-770.

Higgins, S.I. and Cain, M.L., 2002. Spatially realistic plant metapopulation models and the colonization-competition trade-off. J.Ecol., 90: 616-626.

Higgins, S.I., Nathan, R. and Cain, M.L., 2003. Are long-distance dispersal events in plants usually caused by nonstandard means of dispersal? Ecology, 84: 19451956.

Hofmann, E.E., Klinck, J.M., R.A., L., Fach, B. and Murphy, E., 1998. Krill transport in the Scotia sea and environs. Antarct. Sci., 10: 406-415.

Hovestadt, T., Poethke, H.J. and Messner, S., 2000. Variability in dispersal distances generates typical successional patterns: A simple simulation model. Oikos, 90: 612-619.

Hubbell, S.P., 2001. The unified neutral theory of biodiversity and biogeography. Monographs in population biology. Princeton Univ. Press.

Hurtt, G.C. and Pacala, S.W., 1995. The consequences of recruitment limitation: Reconciling chance, history and competitive differences between plants. J.Theor.Biol., 176: 1-12.

Johst, K., Brandl, R. and Eber, S., 2002. Metapopulation persistence in dynamic landscapes: The role of dispersal distance. Oikos, 98: 263-270.

King, A.W. and With, K.A., 2002. Dispersal success on spatially structured landscapes: When do spatial pattern and dispersal behaviour really matter? Ecol. Model., 147: 23-39.

Kneitel, J.M. and Chase, M., 2004. Trade-offs in community ecology: Linking spatial scales and species coexistence. Ecol. Lett., 7: 69-80.

Levin, S.A. and Muller-Landau, H.C., 2000. The emergence of diversity in plant communities. Comptes Rendus de I Academie des Sciences Serie lii-Sciences de la Vie-Life Sciences, 323: 129-139.

Levine, J.M., 2003. A patch modelling approach to the community-level consequences of directional dispersal. Ecology, 84: 1215-1224.

Lockwood, D.R., Hastings, A. and Botsford, L.W., 2002. The effects of dispersal patterns on marine reserves: Does the tail wag the dog? Theor. Popul. Biol., 61: 297-309.

McKane, A.J., Alonso, D. and Sole, R.V., 2004. Analytic solution of Hubbell's model of local community dynamics. Theor. Popul. Biol., 65: 67-73.

Muko, S. and Iwasa, Y., 2000. Species coexistence by permanent spatial heterogeneity in a lottery model. Theor.Popul.Biol., 57: 273-284.

Murrell, D.J., Purves, D.W. and Law, R., 2001. Uniting pattern and process in plant ecology. Trends Ecol. Evol., 16: 529-530.

Nathan, R. and Muller-Landau, H.C., 2000. Spatial patterns of seed dispersal, their determinants and consequences for recruitment. Trends Ecol.Evol., 15: 278285.

Nee, S. and Stone, G., 2003. The end of the beginning for neutral theory. Trends Ecol. Evol., 18: 433-434.

Reed, D.C., Raimondi, P.T., Carr, M.H. and Goldwasser, L., 2000. The role of dispersal and disturbance in determining spatial heterogeneity in sedentary organisms. Ecology, 81: 2011-2026.

Roth, J.K. and Vander Wall, S.B., 2005. Primary and secondary seed dispersal of bush chinquapin (fagaceae) by scatterhoarding rodents. Ecology, 86: 24282439.

Roughgarden, J., Gaines, S. and Possingham, H., 1988. Recruitment dynamics in complex life cycles. Science, 241: 1460-1466. 
Schippers, P. and Jongejans, E., 2005. Release thresholds strongly determine the range of seed dispersal by wind. Ecol. Model., 185: 93-103.

Shorrocks, B., Atkinson, W.D. and Charlesworth, P., 1979. Competition on a divided and ephemeral resource. J. Anim. Ecol., 48: 899-908.

Smith, F. and Witman, J.D., 1999. Species diversity in subtidal landscapes:

Maintenance by physical processes and larval recruitment. Ecology, 80: 5169.

Snyder, R.E. and Chesson, P., 2003. Local dispersal can facilitate coexistence in the presence of permanent spatial heterogeneity. Ecol. Lett., 6: 301-309.

Snyder, R.E. and Chesson, P., 2004. How the spatial scales of dispersal, competition, and environmental heterogeneity interact to affect coexistence. Am. Nat., 164: 633-650.

Stoyan, D. and Wagner, S., 2001. Estimating the fruit dispersion of anemochorous forest trees. Ecol. Model., 145: 35-47.

Tilman, D., 1994. Competition and biodiversity in spatially structured habitats. Ecology, 75: 2-16.

Volkov, I., Banavar, J.R., Hubbell, S.P. and Maritan, A., 2003. Neutral theory and relative species abundance in ecology. Nature, 424: 1035-1037.

Wagner, S., Walder, K., Ribbens, E. and Zeibig, A., 2004. Directionality in fruit dispersal models for anemochorous forest trees. Ecol. Model., 179: 487-498.

Wang, B.C. and Smith, T.B., 2002. Closing the seed dispersal loop. Trends Ecol.Evol., 17: 379-385.

Warren, J. and Topping, C., 2004. A trait specific model of competition in a spatially structured plant community. Ecol. Model., 180: 477-485.

Wehncke, E.V., Hubbell, S.P., Foster, R.B. and Dalling, J.W., 2003. Seed dispersal patterns produced by white-faced monkeys: Implications for the dispersal limitation of neotropical tree species. J. Ecol., 91.

Wenny, D.G., 2001. Advantages of seed dispersal: A re-evaluation of directed dispersal. Evol. Ecol. Res., 3: 51-74.

Wenny, D.G. and Levey, D.J., 1998. Directed seed dispersal by bellbirds in a tropical cloud forest. Proceedings of the National Academy of Sciences of the United States of America, 95: 6204-6207.

Yamazaki, H. and Haury, L.R., 1993. A new lagrangian model to study animal aggregation. Ecol. Model., 69: 99-111.

Yamazaki, H. and Okubo, A., 1995. A simulation of grouping: An aggregating random walk. Ecol. Model., 79: 159-165 